Article

\title{
Evaluation of a New Simplified Inertial Sensor Method against Electrogoniometer for Measuring Wrist Motion in Occupational Studies
}

\author{
Karnica Manivasagam ${ }^{1}$ and Liyun Yang ${ }^{1,2, *(D)}$
}

1 Division of Ergonomics, School of Engineering Sciences in Chemistry, Biotechnology and Health, KTH Royal Institute of Technology, Hälsovägen 11C, SE-141 57 Huddinge, Sweden; karnica@kth.se

2 Unit of Occupational Medicine, Institute of Environmental Medicine, Karolinska Institutet, SE-171 77 Stockholm, Sweden

* Correspondence: liyun.yang@ki.se

\section{check for} updates

Citation: Manivasagam, K.; Yang, L. Evaluation of a New Simplified Inertial Sensor Method against Electrogoniometer for Measuring Wrist Motion in Occupational Studies. Sensors 2022, 22, 1690. https:// doi.org/10.3390/s22041690

Academic Editors: Paolo Perego, Giuseppe Andreoni, Eng Hock Francis Tay, Nicola Francesco Lopomo and Carlo Emilio Standoli

Received: 30 December 2021

Accepted: 17 February 2022

Published: 21 February 2022

Publisher's Note: MDPI stays neutral with regard to jurisdictional claims in published maps and institutional affiliations.

Copyright: (C) 2022 by the authors. Licensee MDPI, Basel, Switzerland. This article is an open access article distributed under the terms and conditions of the Creative Commons Attribution (CC BY) license (https:// creativecommons.org/licenses/by/ $4.0 /)$.

\begin{abstract}
Wrist velocity is an important risk factor for work-related musculoskeletal disorders in the elbow/hand, which is also difficult to assess by observation or self-reports. This study aimed to evaluate a new convenient and low-cost inertial measurement unit (IMU)-based method using gyroscope signals against an electrogoniometer for measuring wrist flexion velocity. Twelve participants performed standard wrist movements and simulated work tasks while equipped with both systems. Two computational algorithms for the IMU-based system, i.e., $\mathrm{IMU}_{\text {norm }}$ and $\mathrm{IMU}_{\text {flex }}$ were used. For wrist flexion/extension, the mean absolute errors (MAEs) of median wrist flexion velocity compared to the goniometer were $<10.1^{\circ} / \mathrm{s}$ for $\mathrm{IMU}_{\text {norm }}$ and $<4.1^{\circ} / \mathrm{s}$ for $\mathrm{IMU}_{\text {flex }}$. During wrist deviation and pronation/supination, all methods showed errors, where the $\mathrm{IMU}_{\text {norm }}$ method had the largest overestimations. For simulated work tasks, the $\mathrm{IMU}_{\text {flex }}$ method had small bias and better accuracy than the $I M U_{\text {norm }}$ method compared to the goniometer, with the MAEs of median wrist flexion velocity $<5.8^{\circ} / \mathrm{s}$. The results suggest that the IMU-based method can be considered as a convenient method to assess wrist motion for occupational studies or ergonomic evaluations for the design of workstations and tools by both researchers and practitioners, and the $\mathrm{IMU}_{\mathrm{flex}}$ method is preferred. Future studies need to examine algorithms to further improve the accuracy of the IMU-based method in tasks of larger variations, as well as easy calibration procedures.
\end{abstract}

Keywords: inertial measurement units; gyroscope; goniometer; wrist flexion velocity; work-related musculoskeletal disorders; ergonomics; hand-intensive work

\section{Introduction}

Work-related musculoskeletal disorders (WMSDs) of the hand/wrist are associated with relatively high medical costs and loss of work days [1]. Repetitive manual work, forceful exertions, prolonged nonneutral postures of the wrist, intensive wrist movements, and hand-arm vibration are identified as critical risk factors for hand/wrist WMSDs, which are common in service industries, manufacturing industries, office work, as well as the healthcare sector [2-8]. From a meta-analysis on the prevalence of WMSDs in Europe's secondary industries during the 21st century, wrist WMSD is among the most common WMSDs with a twelve-month prevalence of $42 \%$ [9].

There are a few observational methods that consider hand/wrist workload and its associated risk [10]. However, the micro-postures and small body parts, including hand/wrist, are shown to be difficult to assess by observations with satisfactory accuracy [10,11]. Poor correlations were reported between the raters using the same observational method on the upper limb, which included the risk factors of frequency and wrist posture [12]. Poor correlations were also found between different observational methods in estimating the hand repetitiveness [13]. Technical measurement methods can provide a quantitative risk 
assessment with good accuracy and reliability, which may contribute to the establishment of exposure-response relationships [14-16]. There are many IMU systems developed for occupational exposure assessment [17]. However, most multi-sensor systems are complex to use in the field by practitioners [18,19]. In addition, many systems can be used for assessments of the upper arm and trunk, but few can be used for assessment of wrist motion [20-22]. Recent research has explored a multi-sensor system focusing on wrist risk assessment. For example, Seidel and colleagues used a multi-sensor system including inertial sensors, a potentiometer, an electromyograph, and a data logger to quantify the hand activity level, showing good accuracy [23]. Still, the wear burden, the required expertise, and the demand of monetary and time resources for such multi-sensor systems can be relatively high.

The importance of assessing wrist angular velocity has been identified and raised by a few authors [24-27]. Researchers could establish a quantitative exposure-response relationship between the wrist flexion velocity and the disorders in the hand/wrist, and an action limit of $20^{\circ}$ / s for median wrist velocity during an $8 \mathrm{~h}$ working day was proposed [25,28]. The electrogoniometer is one commonly used and validated technical measurement method for assessing wrist motion in occupational studies [29]. The proposed action limit on median wrist velocity was also based on measurement data of about 50 occupational groups using electrogoniometers [25]. Although being widely used in research, the electrogoniometer has a few disadvantages of being fragile, expensive, bringing certain burdens to the wearers as it requires cables and a logger to be carried, as well as requiring resources for data analysis.

New generations of Inertial Measurement Units (IMUs) with built-in gyroscopes and Bluetooth technology provide potentials of new methods for assessing wrist velocity [30]. The IMUs can be connected wirelessly to a smartphone application to process the data and generate assessment results automatically, which are convenient for both the wearer, practitioners, and researchers, and at the same time being cheaper and less resource-demanding for data analysis [31,32]. Common IMU systems usually integrate the gyroscopes' and accelerometers' signals to improve the performance of posture and motion assessment under rapid movements [33] where the drift in the gyroscope would be a problem over time and the integration of a magnetometer to correct the drift would be affected by the local magnetic field [34]. It is possible to use the gyroscope directly for velocity assessment, where the gyroscope drift that originates from integration and accumulation of noise will not be a concern. Before such a gyroscope and smartphone application method to be used in the field, the performance of the IMU-based method with various algorithms needs to be evaluated. As the goniometer has been widely used in the field and has laid the foundation for the proposed action limit on wrist velocity at work, it is considered as a standard measure for evaluating the IMU-based method.

Therefore, the aim of this study was to evaluate a new simplified IMU-based method, with two computational algorithms, i.e., the $\mathrm{IMU}_{\text {norm }}$ and the $\mathrm{IMU}_{\mathrm{flex}}$, against an electrogoniometer for measuring wrist flexion velocity during standard wrist movements and simulated work tasks.

\section{Methods}

\subsection{Participants}

Twelve participants (six males and six females, all right-handed) volunteered in the study. The inclusion criteria were healthy adults and pain-free in the dominant wrist/hand. Participants were provided with information about the purpose and design of the study, and signed an informed consent ahead of the experiment. The mean (SD) age of the participants was 26 years (4.1), with a mean height of $169 \mathrm{~cm}(7.3)$, mean weight of $67 \mathrm{~kg}$ (14.9), and mean body mass index of 23.2 (4.3). The study was approved by the Regional Ethics Committee in Stockholm (Dnr, 2019-01206). 


\subsection{Measurement Systems}

A twin axis electrical goniometer (Biometrics Ltd., Newport, UK) with a sampling frequency of $20 \mathrm{~Hz}$ was used as the standard for measuring wrist velocity. The goniometer, with a sampling frequency $52 \mathrm{~Hz}$, was placed on the dorsal surface of the dominant hand with one end-block on the third metacarpal of the hand and the other on the forearm midline, at a distance from the wrist joint where the cables between the two end-blocks were kept straight and not squeezed when the wrist was fully extended. The participants were instructed to hold their arms flat on a table for calibrating the neutral wrist position at the beginning of the experiment (Figure 1).

(a)

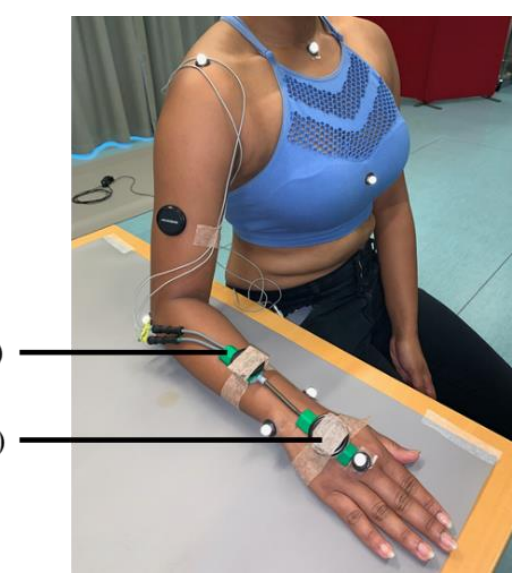

Figure 1. Placement of the goniometer and the two inertial measurement units (IMUs), mounted on the goniometer end-blocks, on (a) the forearm close to the wrist, and (b) the middle of the hand.

Two IMU sensors (Movesense, Suunto, Helsinki) were placed at the top of goniometer end-blocks on both the forearm and the hand (Figure 1a,b). The IMUs were attached using double-sided tape beneath and a medical tape on top to ensure fixation and avoid relative movements. Additionally, a customized wooden block was mounted between the hand IMU and the goniometer end-block for better stability due to the triangle shape of the end-block on the hand. The IMUs were connected and recorded using an open-source iPhone application Movesense showcase v.1.0.5 (Amer Sports Digital Services Oy, Helsinki).

\subsection{Experimental Design}

Before the experiment started, the participants were informed about the tasks that they would perform and were given the opportunity to test and familiarize themselves with the wrist movements. Two types of tests were included in the study to evaluate the IMU-based method against the goniometer: (1) standard wrist movements in three planes, i.e., flexion/extension, radial/ulnar deviation, and pronation/supination, at paces of 30, 60, and 90 beats per minute (bpm), following a metronome (Figure 2a-c); and (2) simulated work tasks, which involved different types of hand/wrist movements: blow-drying hair, folding paper planes, and sorting mail (Figure $2 \mathrm{~d}-\mathrm{f}$ ). Each standard wrist movement was performed for at least 10 cycles and all in a sitting position by a desk. Each work task was performed for one minute at the participants' own chosen pace. The blow-drying hair and sorting mail were performed in a standing position, whilst folding paper planes was performed in a sitting position. 

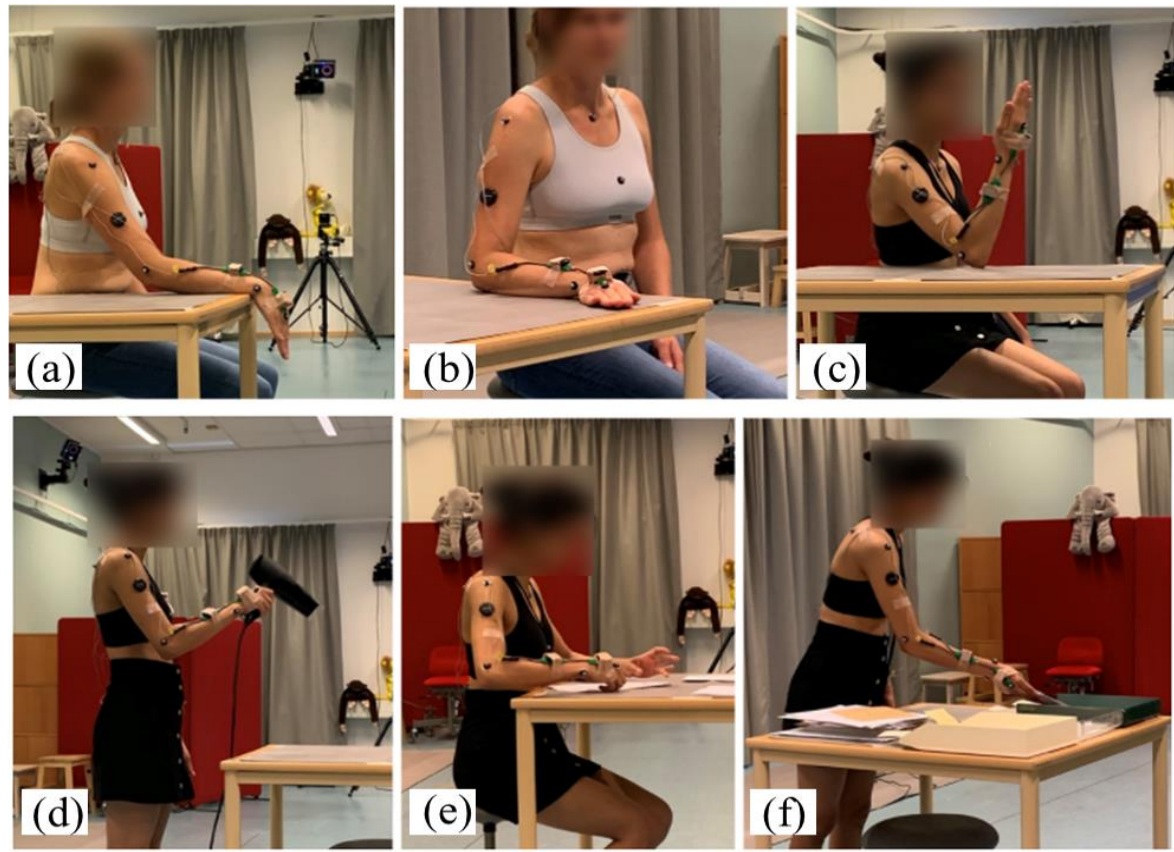

Figure 2. Three standard wrist movements including: (a) flexion/extension, (b) radial/ulnar deviation, and (c) pronation/supination; and three simulated work tasks including: (d) blow-drying hair, (e) folding paper planes, and (f) sorting mail.

\subsection{Signal Processing and Statistical Analysis}

The measurement data from both the systems were processed in MATLAB (2021, MathWorks, Inc., Natick, MA, USA). For the goniometer, the flexion/extension angle output was low-pass-filtered at $5 \mathrm{~Hz}$ [35] and the derivatives of the flexion/extension angle were calculated as wrist flexion velocity. For the IMUs, the output from the gyroscopes in three axes of both IMUs on the hand and forearm were synchronized, low-pass-filtered (4thorder Butterworth filter with a cut-off frequency at $5 \mathrm{~Hz}$ ), and down-sampled to $20 \mathrm{~Hz}$. Two computational algorithms were used for calculating the wrist flexion velocity: $\mathrm{IMU}_{\text {norm }}$ and $\mathrm{IMU}_{\text {flex }}$. For $\mathrm{IMU}_{\text {norm, }}$, the vector norm of gyroscopes of both the hand and forearm IMUs were calculated and then subtracted, and the absolute value of the difference was taken as the wrist velocity as follows:

$v_{\text {wrist }}=\left|\sqrt{\left(g_{\text {xhand }}\right)^{2}+\left(g_{\text {yhand }}\right)^{2}+\left(g_{\text {zhand }}\right)^{2}}-\sqrt{\left(g_{\text {xforearm }}\right)^{2}+\left(g_{\text {yforearm }}\right)^{2}+\left(g_{\text {zforearm }}\right)^{2}}\right|$

For $\mathrm{IMU}_{\text {flex }}$, the gyroscope output at the $\mathrm{x}$-axis (i.e., the flexion/extension axis) of the hand and forearm IMUs were subtracted as the wrist velocity as follows:

$$
v_{\text {wrist }}=\sqrt{\left(g_{\text {xhand }}-g_{\text {forearm }}\right)^{2}}
$$

The calculated IMU-based wrist velocities were synchronized with the goniometer output, and the 10th, 50th, and 90th percentiles of the wrist flexion velocity were calculated for each of the standard movements and simulated work tasks. The two computational algorithms for IMUs were compared against the goniometer separately. The mean absolute errors (MAEs) and its standard deviation (SD) were computed between each pair of comparison for all tasks at the 10th, 50th, and 90th percentile. In addition, correlation plots and Bland-Altman plots of the median wrist flexion velocity for all tasks were used to compare the $I M U_{\text {norm }}$ and $I M U_{\text {flex }}$ against the goniometer. 


\section{Results}

The measured wrist flexion velocity by the goniometer and two IMU-based methods and the mean absolute errors (MAEs) from the comparison during standard wrist movements are shown in Table 1. For the wrist flexion/extension, the $\mathrm{IMU}_{\text {flex }}$ had good accuracy with the MAEs ranging from $1.4^{\circ} / \mathrm{s}$ to $4.1^{\circ} / \mathrm{s}$ for median wrist velocity as the pace increased. The $\mathrm{IMU}_{\text {norm }}$ had slightly larger errors with the MAEs of median wrist velocity ranging from $4.1^{\circ} / \mathrm{s}$ up to $10.1^{\circ} \mathrm{s}$. Larger errors were observed for the 90th percentile of wrist velocity between the IMU-based methods and goniometer as the speed increased.

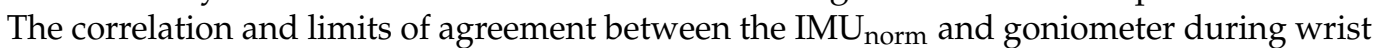
flexion/extension are shown in Figure $3 a, b$, and those between the $\mathrm{IMU}_{\text {flex }}$ and goniometer are shown in Figure $3 \mathrm{c}, \mathrm{d}$. The squared correlation coefficient $\left(\mathrm{r}^{2}\right)$ was high between both IMU-based methods and the goniometer $\left(\mathrm{r}^{2}=0.98\right.$ and 1.00 , respectively). The $\mathrm{IMU}_{\text {norm }}$ had a tendency of underestimation when the flexion speed increased from 30 to 90 BPM, with limits of agreement of $-20^{\circ} / \mathrm{s}$ and $6.4^{\circ} / \mathrm{s}$.

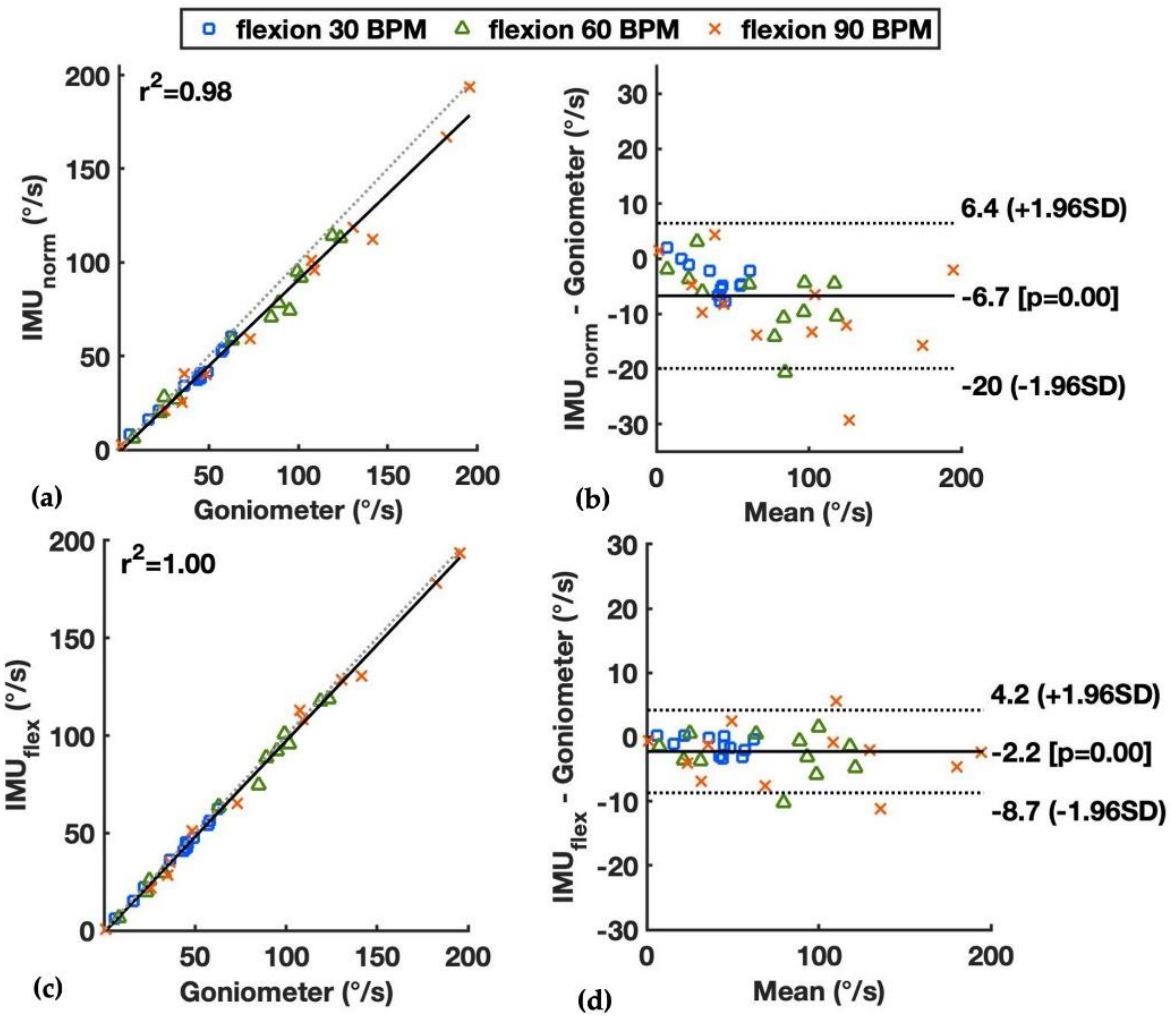

Figure 3. Median wrist flexion velocity during wrist flexion/extension at paces of 30,60, and 90 BPM measured by the goniometer and the IMU-based methods, showing by linear correlation and BlandAltman plots: $(\mathbf{a}, \mathbf{b})$ the $I M U_{\text {norm }}$ and goniometer; and $(\mathbf{c}, \mathbf{d})$ the $\mathrm{IMU}_{\mathrm{flex}}$ and goniometer. Squared correlation coefficients $\left(\mathrm{r}^{2}\right)$ and limits of agreements are presented.

During wrist deviation and pronation/supination, the flexion velocity should theoretically be close to zero. However, errors were observed in the goniometer and two IMU-based methods, where the $\mathrm{IMU}_{\text {norm }}$ had the largest overestimations with the mean values of the 50th percentile of flexion velocity up to $23.4^{\circ} / \mathrm{s}$ for deviation at $90 \mathrm{BPM}$ and $51.4^{\circ} / \mathrm{s}$ for pronation/supination at 90 BPM (see Individual Method, Table 1). The correlation and limits of agreement between the $\mathrm{IMU}_{\text {norm }}$ and goniometer during wrist deviation are shown in Figure $4 \mathrm{a}, \mathrm{b}$, and those during pronation/supination are shown in Figure 5a,b. Distinctive differences were observed between the $\mathrm{IMU}_{\text {norm }}$ and goniometer, with larger differences as the speed of motion increased from 30 to 90 BPM, and larger differences during the pronation/supination than deviation. The $\mathrm{IMU}_{\mathrm{flex}}$ and goniometer 
had errors calculating the wrist flexion velocity at similar levels during deviation, with mean values of the 50th percentile wrist velocity up to $8.1^{\circ} / \mathrm{s}$ and $7.9^{\circ} / \mathrm{s}$, respectively (see Individual Method, Table 1). For wrist pronation/supination, the $\mathrm{IMU}_{\text {flex }}$ had smaller errors compared to the goniometer (mean values of 50th percentile wrist velocity up to $9.6^{\circ} / \mathrm{s}$ vs. $19.1^{\circ} / \mathrm{s}$, respectively). The correlation and limits of agreement between the $\mathrm{IMU}_{\text {flex }}$ and goniometer during wrist deviation are shown in Figure $4 \mathrm{c}, \mathrm{d}$, and those during pronation/supination are shown in Figure $5 \mathrm{c}, \mathrm{d}$. As the values of the goniometer during deviation and pronation/supination were shown to be flawed, the difference between the IMU-based methods and the goniometer in these two wrist movements should not be considered as errors compared to the ground truth.

Table 1. The mean and standard deviation (SD) of wrist flexion velocity from the goniometer, the $\mathrm{IMU}_{\text {norm }}$ and the $\mathrm{IMU}_{\mathrm{flex}}$, and the mean absolute errors (MAEs) and SD for the comparisons of methods during the standard wrist movements at paces of 30,60, and 90 beats per minute (BPM). Data are shown for the 10th, 50th, and 90th percentiles of the wrist velocity with 12 participants included.

\begin{tabular}{|c|c|c|c|c|c|c|}
\hline \multirow{2}{*}{$\%$} & \multirow{2}{*}{ Percentile } & \multicolumn{3}{|c|}{ Individual Method, Mean (SD) } & \multicolumn{2}{|c|}{ Comparison of Methods, MAEs (SD) } \\
\hline & & Goniometer & $\mathrm{IMU}_{\text {norm }}$ & $\mathrm{IMU}_{\text {flex }}$ & $\mathrm{IMU}_{\text {norm}}-$ Goniometer & $\mathrm{IMU}_{\text {flex }}-$ Goniometer \\
\hline \multicolumn{7}{|c|}{ Flexion/Extension } \\
\hline \multirow[t]{3}{*}{$30 \mathrm{BPM}$} & 10th & $4.2(3.2)$ & $4.8(2.2)$ & $4.2(3.1)$ & $1.4(0.9)$ & $0.3(0.2)$ \\
\hline & 50 th & $40.7(17.5)$ & $36.9(15.5)$ & $39.4(16.9)$ & $4.1(2.6)$ & $1.4(1.2)$ \\
\hline & 90th & $125.7(52.0)$ & $116.8(48.5)$ & $118.6(47.2)$ & $9.0(4.2)$ & $7.5(6.4)$ \\
\hline \multirow[t]{3}{*}{$60 \mathrm{BPM}$} & 10th & $4.0(4.7)$ & $4.3(3.3)$ & $4.0(5.1)$ & $1.3(0.8)$ & $0.4(0.5)$ \\
\hline & 50 th & $72.1(40.2)$ & $64.8(37.0)$ & $69.4(39.7)$ & $7.8(5.5)$ & $3.1(2.8)$ \\
\hline & 90th & $233.1(102.6)$ & $217.9(98.0)$ & $219.2(92.3)$ & $15.2(7.5)$ & $14.3(12.1)$ \\
\hline \multirow[t]{3}{*}{$90 \mathrm{BPM}$} & 10th & $6.6(9.2)$ & $5.4(6.5)$ & $6.8(10.5)$ & $2.0(3.0)$ & $1.0(1.4)$ \\
\hline & 50 th & $90.6(63.7)$ & $81.4(60.1)$ & $87.8(63.3)$ & $10.1(7.7)$ & $4.1(3.2)$ \\
\hline & 90th & $322.0(145.7)$ & $300.8(143.3)$ & $301.8(136.7)$ & $21.4(12.1)$ & $20.2(14.1)$ \\
\hline \multicolumn{7}{|c|}{ Radial/Ulnar Deviation } \\
\hline \multirow[t]{3}{*}{$30 \mathrm{BPM}$} & 10th & $0.5(0.2)$ & $1.4(0.6)$ & $0.5(0.2)$ & $0.9(0.5)$ & $0.1(0.1)$ \\
\hline & 50 th & $4.2(1.6)$ & $12.0(6.1)$ & $3.7(1.5)$ & $7.9(5.2)$ & $0.8(0.7)$ \\
\hline & 90th & $16.9(7.1)$ & $51.2(19.2)$ & $16.1(7.4)$ & $34.3(16.1)$ & $2.9(2.9)$ \\
\hline \multirow[t]{3}{*}{$60 \mathrm{BPM}$} & 10th & $0.9(0.5)$ & $1.7(0.8)$ & $0.8(0.3)$ & $0.9(0.7)$ & $0.2(0.2)$ \\
\hline & 50th & $7.9(4.3)$ & $22.3(13.2)$ & $7.2(3.4)$ & $14.4(11.9)$ & $1.4(1.9)$ \\
\hline & 90th & $37.0(35.9)$ & $101.2(35.0)$ & $39.1(35.7)$ & $65.1(32.5)$ & $6.8(7.6)$ \\
\hline \multirow[t]{3}{*}{$90 \mathrm{BPM}$} & 10th & $0.9(0.7)$ & $2.0(1.2)$ & $0.9(0.7)$ & $1.1(0.7)$ & $0.2(0.1)$ \\
\hline & 50th & $7.9(3.7)$ & $23.4(18.0)$ & $8.1(4.1)$ & $15.7(14.9)$ & $1.3(1.2)$ \\
\hline & 90th & $33.5(16.0)$ & $130.0(55.5)$ & $38.5(22.3)$ & $98.5(47.9)$ & $9.0(12.4)$ \\
\hline \multicolumn{7}{|c|}{ Pronation/Supination } \\
\hline \multirow[t]{3}{*}{$30 \mathrm{BPM}$} & 10 th & $1.1(0.5)$ & $1.3(0.8)$ & $0.7(0.2)$ & $0.3(0.3)$ & $0.5(0.3)$ \\
\hline & 50 th & $10.2(5.0)$ & $26.2(10.1)$ & $5.0(1.3)$ & $16.1(8.1)$ & $5.2(4.5)$ \\
\hline & 90th & $39.5(15.4)$ & $77.2(29.2)$ & $18.9(6.6)$ & $37.7(23.8)$ & $20.6(12.8)$ \\
\hline \multirow[t]{3}{*}{$60 \mathrm{BPM}$} & 10th & $1.6(1.0)$ & $1.9(1.9)$ & $1.2(0.7)$ & $0.7(1.0)$ & $0.5(0.4)$ \\
\hline & 50th & $18.5(10.0)$ & $46.8(23.1)$ & $10.1(7.4)$ & $29.1(19.0)$ & $8.6(8.3)$ \\
\hline & 90th & $103.6(100.8)$ & $170.1(89.6)$ & $61.3(105.3)$ & $69.7(49.3)$ & $42.3(26.7)$ \\
\hline \multirow[t]{3}{*}{$90 \mathrm{BPM}$} & 10th & $1.6(1.5)$ & $2.0(2.0)$ & $1.0(0.7)$ & $0.6(0.9)$ & $0.7(0.9)$ \\
\hline & 50th & $19.1(10.5)$ & $51.4(32.7)$ & $9.6(5.0)$ & $32.3(24.7)$ & $9.5(6.4)$ \\
\hline & 90th & $101.4(54.4)$ & $197.3(83.6)$ & $41.3(17.2)$ & $96.6(50.8)$ & $60.1(45.7)$ \\
\hline
\end{tabular}

The wrist flexion velocity measured by the goniometer and two IMU-based methods and the mean absolute errors (MAEs) during simulated work tasks are shown in Table 2. The $\mathrm{IMU}_{\text {flex }}$ had an overall better accuracy than the $\mathrm{IMU}_{\text {norm }}$ compared to the goniometer, with the MAEs of median wrist flexion velocity $<5.8^{\circ} / \mathrm{s}$ for all work tasks, vs. $<10.6^{\circ} / \mathrm{s}$ for $\mathrm{IMU}_{\text {norm }}$ (see Comparison of Methods, Table 2). The largest MAEs were observed during blow-drying hair for both IMU-based methods, whereas the smallest differences were observed during folding paper planes. The correlation and limits of agreement between 
the $\mathrm{IMU}_{\text {norm }}$ and goniometer during three work tasks are shown in Figure $6 \mathrm{a}, \mathrm{b}$, and those between the IMU flex and goniometer are shown in Figure 6c,d. The IMU flex had a high squared correlation coefficient compared to the goniometer $\left(r^{2}=0.93\right)$, a small bias of $0.78^{\circ} / \mathrm{s}$, and limits of agreement of $-10^{\circ} / \mathrm{s}$ and $12^{\circ} / \mathrm{s}$. The $\mathrm{IMU}_{\text {norm }}$ had a lower squared correlation coefficient compared to the goniometer $\left(\mathrm{r}^{2}=0.71\right)$, a bias of $-3^{\circ} / \mathrm{s}$, and larger limits of agreement of $-26^{\circ} / \mathrm{s}$ and $20^{\circ} / \mathrm{s}$.

Table 2. The mean and standard deviation (SD) of wrist flexion velocity from the goniometer, the $\mathrm{IMU}_{\text {norm }}$ and the $\mathrm{IMU}_{\mathrm{flex}}$, and the mean absolute errors (MAEs) and SD for the comparisons of methods during the simulated work tasks. Data are shown for the 10th, 50th, and 90th percentiles of the wrist velocity during each task with 12 participants included.

\begin{tabular}{|c|c|c|c|c|c|c|}
\hline \multirow{2}{*}{$\% / \mathrm{s}$} & \multirow{2}{*}{ Percentile } & \multicolumn{3}{|c|}{ Individual Method, Mean (SD) } & \multicolumn{2}{|c|}{ Comparison of Methods, MAEs (SD) } \\
\hline & & Goniometer & IMU $_{\text {norm }}$ & $\mathrm{IMU}_{\text {flex }}$ & $\mathrm{IMU}_{\text {norm }}-$ Goniometer & $\mathrm{IMU}_{\text {flex }}-$ Goniometer \\
\hline \multirow{3}{*}{$\begin{array}{c}\text { Blow-drying } \\
\text { hair }\end{array}$} & 10th & $5.2(4.0)$ & $5.8(4.2)$ & $6.6(4.5)$ & $1.4(1.7)$ & $1.4(1.6)$ \\
\hline & 50 th & $36.3(29.9)$ & $37.8(24.0)$ & $40.5(28.4)$ & $10.6(12.7)$ & $5.8(6.0)$ \\
\hline & 90th & $111.7(58.8)$ & $105.9(44.3)$ & $115.6(54.9)$ & $24.6(26.0)$ & $10.8(10.3)$ \\
\hline \multirow{3}{*}{$\begin{array}{l}\text { Folding paper } \\
\text { planes }\end{array}$} & 10th & $3.1(1.0)$ & $2.6(1.0)$ & $2.9(1.1)$ & $0.6(0.5)$ & $0.3(0.2)$ \\
\hline & 50 th & $23.1(5.9)$ & $18.9(6.2)$ & $22.0(6.0)$ & $4.4(2.3)$ & $1.6(1.2)$ \\
\hline & 90th & 93.0 (18.7) & 74.9 (21.9) & $92.0(22.7)$ & $19.1(9.5)$ & $7.9(5.1)$ \\
\hline \multirow{3}{*}{ Sorting mail } & 10th & $8.9(1.5)$ & $7.4(2.4)$ & $8.5(1.7)$ & $2.2(1.6)$ & $0.8(0.4)$ \\
\hline & 50th & $51.8(9.1)$ & $45.6(9.8)$ & $51.1(8.9)$ & $8.1(8.4)$ & $3.6(2.9)$ \\
\hline & 90th & $145.4(19.9)$ & $122.4(21.8)$ & $140.5(23.0)$ & $25.8(13.5)$ & $7.9(6.5)$ \\
\hline
\end{tabular}
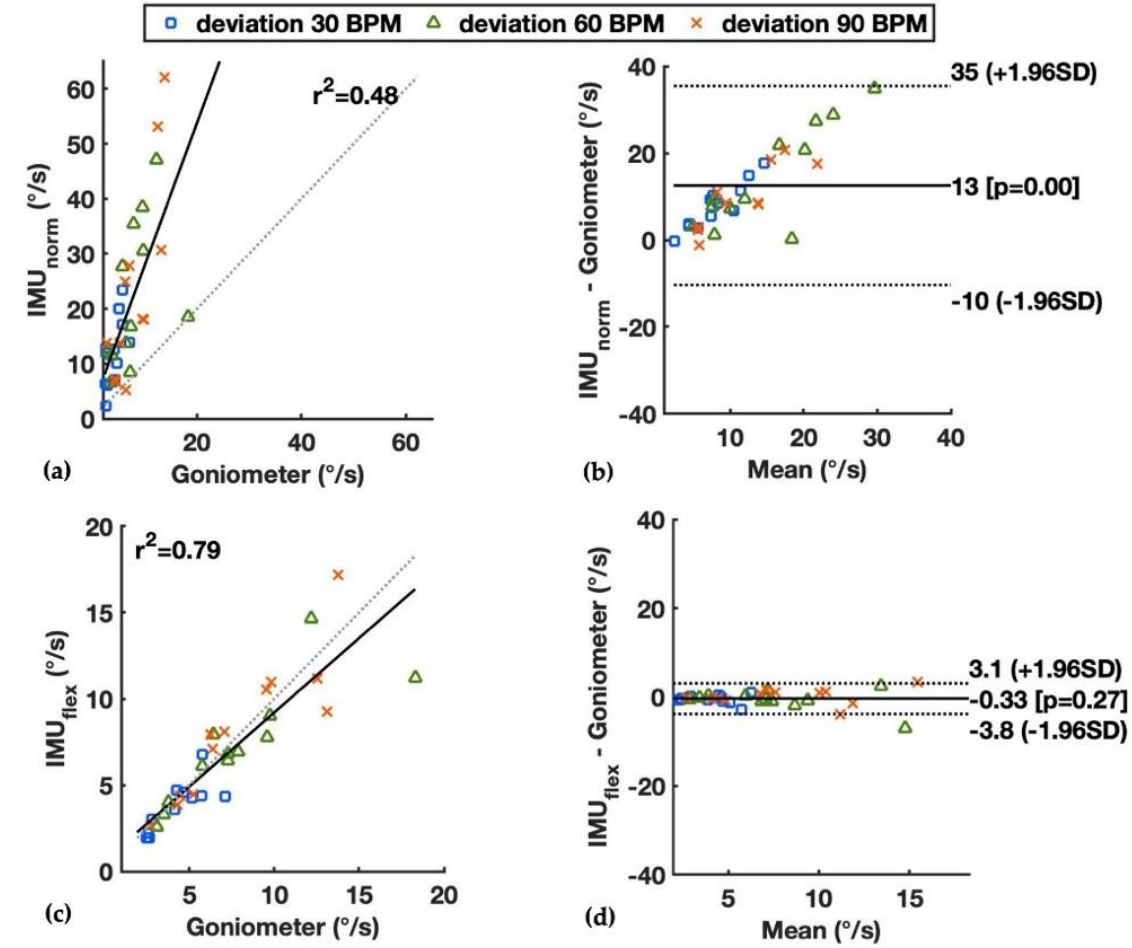

Figure 4. Median wrist flexion velocity during wrist deviation at paces of 30,60, and 90 BPM measured by the goniometer and the IMU-based methods, showing by linear correlation and BlandAltman plots: $(\mathbf{a}, \mathbf{b})$ the $\mathrm{IMU}_{\text {norm }}$ and goniometer; and $(\mathbf{c}, \mathbf{d})$ the $\mathrm{IMU}_{\mathrm{flex}}$ and goniometer. Squared correlation coefficients $\left(\mathrm{r}^{2}\right)$ and limits of agreements are presented. 
- pronation/supination $30 \mathrm{BPM} \triangle$ pronation/supination $60 \mathrm{BPM} \times$ pronation/supination $90 \mathrm{BPM}$
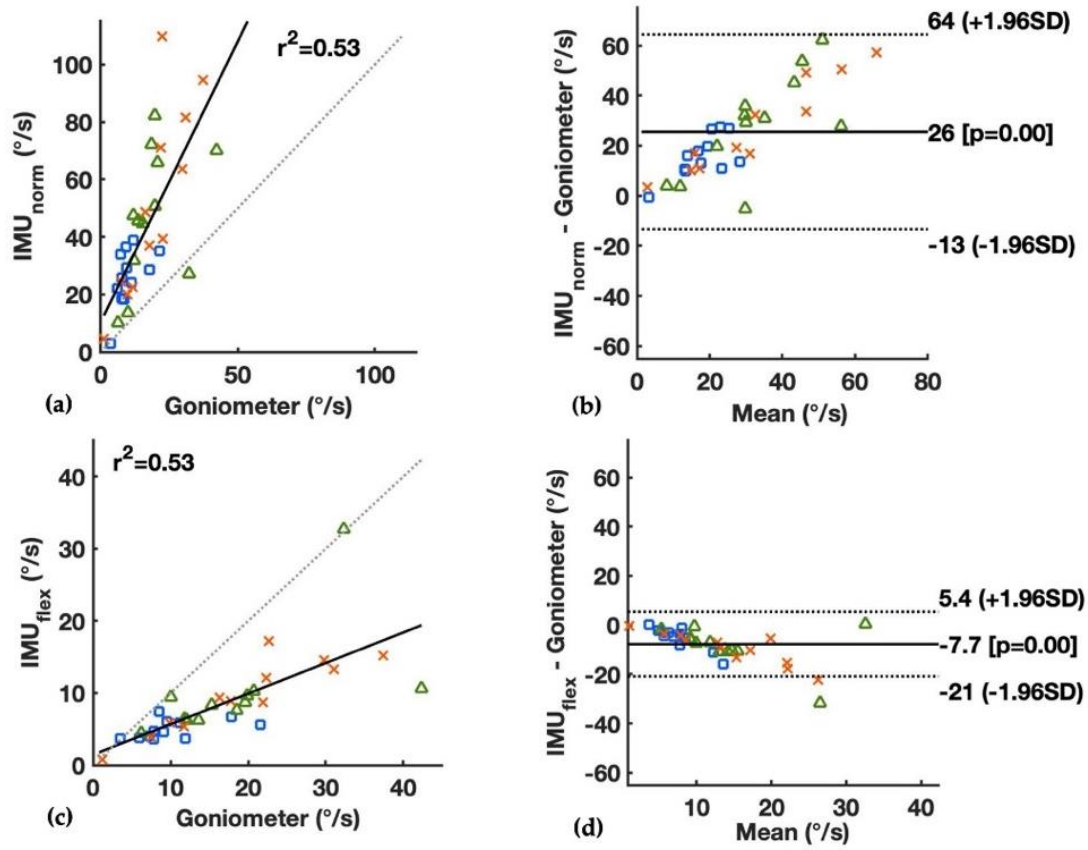

Figure 5. Median wrist flexion velocity during wrist pronation/supination at paces of 30,60, and 90 BPM measured by the goniometer and the IMU-based methods, showing by linear correlation and Bland-Altman plots: $(\mathbf{a}, \mathbf{b})$ the $\mathrm{IMU}_{\text {norm }}$ and goniometer; and $(\mathbf{c}, \mathbf{d})$ the $\mathrm{IMU}_{\text {flex }}$ and goniometer. Squared correlation coefficients $\left(\mathrm{r}^{2}\right)$ and limits of agreements are presented.

\section{0 hair blow drying $\Delta$ paper plane folding $\times$ mail sorting}
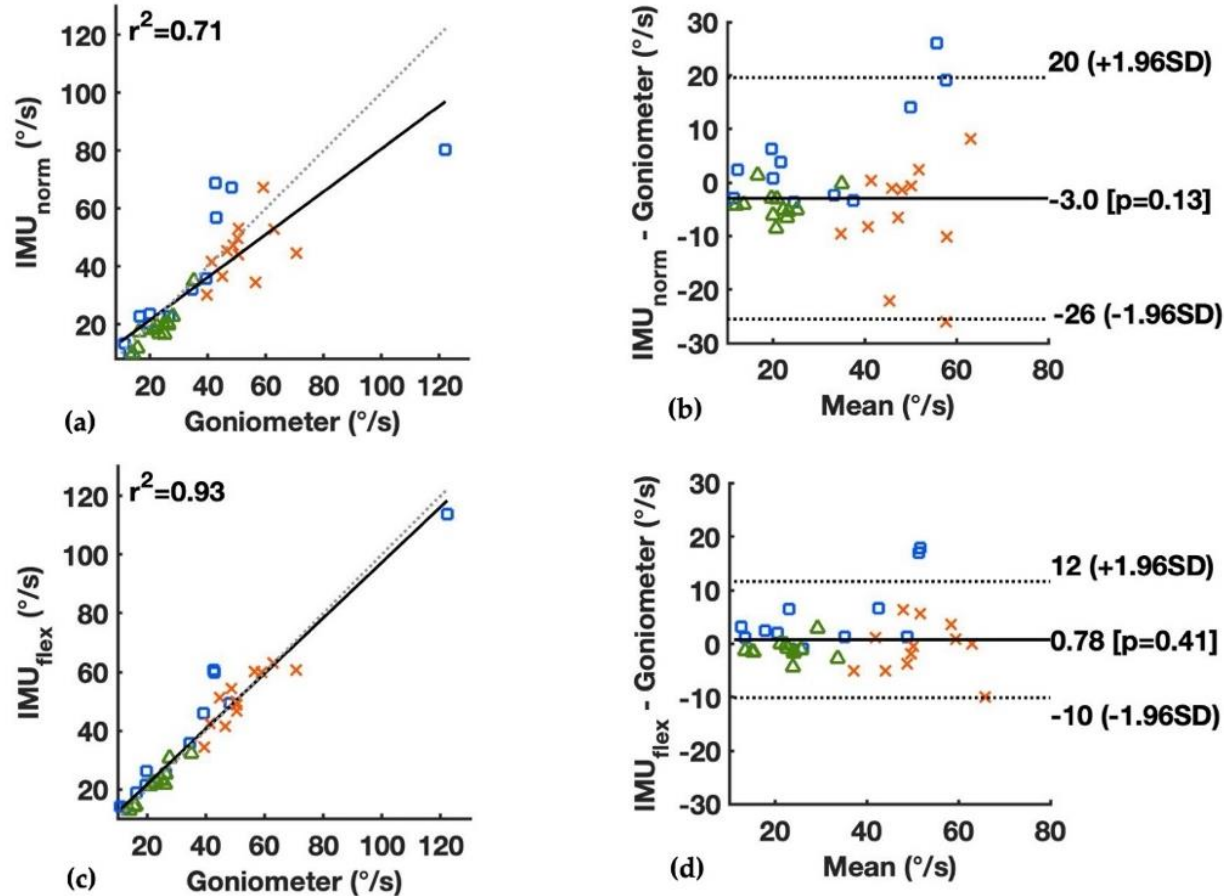

Figure 6. Median wrist flexion velocity for three simulated work tasks measured by the goniometer and the IMU-based methods, showing by linear correlation and Bland-Altman plots: $(\mathbf{a}, \mathbf{b})$ the $\mathrm{IMU}_{\text {norm }}$ and goniometer; and $(\mathbf{c}, \mathbf{d})$ the $\mathrm{IMU}_{\text {flex }}$ and goniometer. Squared correlation coefficients $\left(\mathrm{r}^{2}\right)$ and limits of agreements are presented. 


\section{Discussion}

This study evaluated a new simplified IMU-based method using two computational algorithms, i.e., the $\mathrm{IMU}_{\text {norm }}$ and the $\mathrm{IMU}_{\text {flex, }}$ against the electrogoniometer for measuring wrist flexion velocity during standard wrist movements and simulated work tasks. The results showed that the $\mathrm{IMU}_{\mathrm{flex}}$ method had an overall better performance than the $\mathrm{IMU}_{\text {norm }}$ compared to the goniometer in all standard wrist movements and simulated work tasks, with relatively small bias. The new IMU-based method has a great potential to be applied as a convenient method for risk assessment of wrist motion at work by both researchers and practitioners.

The standard wrist movements including motion in three planes were chosen to test the performance of the IMU-based method compared to the goniometer under constrained conditions. During standard wrist flexion/extension movements, it was observed that the $\mathrm{IMU}_{\text {flex }}$ had high agreement and small bias compared with the goniometer method (Table 1 and Figure 3 ). The IMU $\mathrm{I}_{\text {norm }}$ method had slightly larger differences compared to the goniometer, especially when the speed increased from 30 to 90 BPM.

For the radial-ulnar deviation movements, the wrist flexion velocity should theoretically be close to zero. However, errors were observed both by the goniometer and the IMU-based methods. The measured flexion velocity, which deviated from zero by all methods, could partly be explained by the coupling of wrist flexion-extension and deviation [36]. Therefore, there were wrist movements in the flexion-extension plane even though the participants were instructed to only move in the radial-ulnar deviation plane, which are mainly due to the constraints of carpal ligaments and muscle contraction patterns [36]. In addition, the errors of the IMU-based methods could be caused by the unaligned deviation and rotation axes of the built-in gyroscopes of the hand and forearm IMUs, and the soft tissue artifacts, i.e., the relative movement between the skin and underlying bone. For the $\mathrm{IMU}_{\text {norm }}$ method, the extra high errors were partly expected (Table 1 and Figures 4 and 5), as the inclusion of all three axes of the gyroscopes would also record the deviation and rotation movements of the wrist. For the $\mathrm{IMU}_{\mathrm{flex}}$ method, as the flexion axes of the gyroscopes of the hand and forearm IMUs were assumed to be aligned, estimation errors would occur when the axes were not perfectly aligned. The misalignment could happen due to the irregular articular surface of the carpal bones, the placement of the two IMUs on goniometer end-blocks, and combined wrist movements on more than two planes. For the goniometer, the errors can be explained by its inherent crosstalk between the flexion and deviation recordings due to twisting of the goniometer transducer, the impact of forearm rotation, and the movement of skin $[35,37,38]$, which could be observed during the deviation and supination/pronation movements (see Individual method, Table 1). Nevertheless, the $\mathrm{IMU}_{\text {flex }}$ method showed small bias in the wrist flexion velocity results compared with the goniometer during deviation (Table 1 and Figure 4c,d). For wrist pronation/supination, the $\mathrm{IMU}_{\text {flex }}$ had smaller errors compared to the goniometer, i.e., smaller values above zero in this case (Table 1 and Figure $5 c, d$ ).

Three tasks using the hand/wrist in different intensities were included in the experiment. The task of folding paper planes was the least intensive and showed the smallest wrist velocities measured by all three methods. It also had the smallest differences between the two IMU-based methods and goniometer (see Comparison of methods, Table 2). Blowdrying hair and sorting mail involved more intensive wrist motions and combined motions on different planes of the wrist joint. The task of sorting mail showed the highest wrist velocities measured by all three methods, with the mean 50th percentile of wrist velocity between 45.6 and $51.8^{\circ}$ / s (see Individual method, Table 2). The task of blow-drying hair had a larger variation between the participants, as they were instructed to perform the task at their own chosen pace. Overall, the $\mathrm{IMU}_{\text {flex }}$ method had smaller MAEs than the $\mathrm{IMU}_{\text {norm }}$ in comparison with the goniometer during all simulated work tasks. 


\section{Limitations and Future Studies}

As discussed above, during the radial/ulnar deviation and pronation/supination movements, the recorded wrist flexion velocity should theoretically be close to zero. Nonetheless, the goniometer, which is considered as a standard measure in this study, showed errors. Therefore, the difference between the goniometer and two IMU-based methods cannot be considered as errors compared to the ground truth. Still, the crosstalk was shown to have a marginal effect on the assessment when the wrist flexion summary measures were used in the occupational studies [37,39]. In addition, to be used for risk assessment in occupational studies with the proposed action level of wrist velocity [28], the comparison of the IMU-based methods against the goniometer is still valuable. Future work will evaluate both the IMU-based methods and the goniometer against an optical motion tracking system, which can be considered as a standard measurement in a laboratory environment.

Another limitation was that in order to equip both systems on participants, due to lack of space on the hand and forearm, the IMUs were mounted on the goniometer end-block, which was not ideal. When used by itself for measuring wrist motion, the IMUs would be placed on the skin, and the forearm IMU would be closer to the wrist joint. In this way, the two IMUs can be better aligned, and the impact of soft tissue artifacts can be reduced, especially during pronation/supination.

In the current study, no extra calibration procedure was performed to align the gyroscope axes, as the IMUs were mounted on the hand and forearm so the text on both IMU cases were aligned. The two IMU-based algorithms could be compared to a goniometer and showed sufficient accuracy in simulated work tasks. One could expect that when using in the field studies, the two IMUs may be misaligned due to errors or the relative movement of sensors on the skin, and calibration would be crucial to reduce the errors. Therefore, simple calibration procedures need to be explored and evaluated for future field studies in the next step.

In addition, in the field where exposures of a whole working day are measured, the variation in the hand/wrist movements will be higher compared to the laboratory setting. The sample size of this study was small, but for similar studies on wrist motion with human participants, a sample size of 10 to 12 was common $[36,40]$.

Future studies need to examine algorithms to improve the accuracy of the IMUbased method, especially in tasks involving combined movements of wrist deviation and pronation/supination and movements of larger variation. Potential calibration procedures can be explored to improve the alignment of gyroscope axes of the two IMUs, without adding too much complexity to the method for being used by practitioners. It is also of great interest to investigate the performance of the IMU-based method in field studies. When the accuracy is of highest need, sophisticated measurement methods such as an optical motion tracking system can be used in the laboratory, while for occupational studies in the field, or for studies evaluating new work designs and tools involving hand-intensive work, the IMU-based method has the great advantage of being easy to use, having a low wearer burden, being cost-efficient, and providing direct risk assessment results.

\section{Conclusions}

A new simplified IMU-based method using two computational algorithms, i.e., the $\mathrm{IMU}_{\text {norm }}$ and the $\mathrm{IMU}_{\text {flex }}$, was evaluated against the goniometer for measuring wrist flexion velocity in this study. The IMU flex method showed small bias in small- to medium-paced standard hand/wrist movements and all simulated work tasks compared to the goniometer. The results suggest that the IMU-based method has great potential to be used for risk assessments of wrist motion by both researchers and practitioners, in occupational studies, and in design and evaluating new workstations or tools. Future work should look into algorithms to improve the accuracy of the IMU-based method in tasks involving combined movements of wrist deviation and pronation/supination, as well as easy calibration procedures for field studies. 
Author Contributions: Conceptualization, L.Y.; methodology, K.M. and L.Y.; formal analysis, K.M. and L.Y.; data curation, K.M. and L.Y.; writing-original draft preparation, K.M.; writing-review and editing, K.M. and L.Y.; supervision, L.Y.; project administration, L.Y.; funding acquisition, L.Y. All authors have read and agreed to the published version of the manuscript.

Funding: This research was funded by the Swedish Research Council for Health Working Life and Welfare (FORTE), grant number 2017-01209.

Institutional Review Board Statement: The study was conducted according to the guidelines of the Declaration of Helsinki, and approved by the Regional Ethics Committee in Stockholm, Sweden (Dnr: 2019-01206).

Informed Consent Statement: Informed consent was obtained from all participants involved in the study.

Data Availability Statement: Data presented in the paper are available on request from the corresponding author L.Y.

Acknowledgments: The authors are grateful to Mikael Forsman for invaluable support in the project administration and funding acquisition, and to all participants who volunteered in the study.

Conflicts of Interest: The authors declare no conflict of interest. The funders had no role in the design of the study; in the collection, analyses, or interpretation of data; in the writing of the manuscript, or in the decision to publish the results.

\section{References}

1. Bureau of Labor Statistics. Nonfatal Occupational Injuries and Illnesses Requiring Days Away from Work, 2015. 2016. Available online: https:/ / www.bls.gov/news.release/pdf/osh2.pdf (accessed on 29 December 2021).

2. Barr, A.E.; Barbe, M.F.; Clark, B.D. Work-related musculoskeletal disorders of the hand and wrist: Epidemiology, pathophysiology, and sensorimotor changes. J. Orthop. Sports Phys. Ther. 2004, 34, 610-627. [CrossRef] [PubMed]

3. PKeir, J.; Zuniga, A.F.; Mulla, D.M.; Somasundram, K.G. Relationships and Mechanisms Between Occupational Risk Factors and Distal Upper Extremity Disorders. Hum. Factors 2021, 63, 5-31. [CrossRef]

4. van Rijn, R.M.; Huisstede, B.M.A.; Koes, B.W.; Burdorf, A. Associations between work-related factors and the carpal tunnel syndrome-A systematic review. Scand. J. Work. Environ. Health 2009, 35, 19-36. [CrossRef]

5. Muggleton, J.M.; Allen, R.; Chappell, P.H. Hand and arm injuries associated with repetitive manual work in industry: A review of disorders, risk factors and preventive measures. Ergonomics 1999, 42, 714-739. [CrossRef] [PubMed]

6. Bernard, B.P.; Putz-Anderson, V. Musculoskeletal Disorders and Workplace Factors A Critical Review of Epidemiologic Evidence for Work-Related Musculoskeletal Disorders of the Neck, Upper Extremity, and Low Back. 1997. Available online: http: //www.cdc.gov/niosh/docs/97-141/ (accessed on 29 December 2021).

7. $\quad$ Epstein, S.; Sparer, E.H.; Tran, B.N.; Ruan, Q.Z.; Dennerlein, J.T.; Singhal, D.; Lee, B.T. Prevalence of Work-Related Musculoskeletal Disorders Among Surgeons and Interventionalists: A Systematic Review and Meta-analysis. JAMA Surg. 2018, 153 , e174947. [CrossRef] [PubMed]

8. Kozak, A.; Schedlbauer, G.; Wirth, T.; Euler, U.; Westermann, C.; Nienhaus, A. Association between work-related biomechanical risk factors and the occurrence of carpal tunnel syndrome: An overview of systematic reviews and a meta-analysis of current research. BMC Musculoskelet. Disord. 2015, 16, 231. [CrossRef] [PubMed]

9. Govaerts, R.; Tassignon, B.; Ghillebert, J.; Serrien, B.; De Bock, S.; Ampe, T.; El Makrini, I.; Vanderborght, B.; Meeusen, R.; De Pauw, K. Prevalence and incidence of work-related musculoskeletal disorders in secondary industries of 21st century Europe: A systematic review and meta-analysis. BMC Musculoskelet. Disord. 2021, 22, 1-30. [CrossRef] [PubMed]

10. Takala, E.P.; Pehkonen, I.; Forsman, M.; Hansson, G.Å.; Mathiassen, S.E.; Neumann, W.P.; Sjøgaard, G.; Veiersted, K.B.; Westgaard, R.H.; Winkel, J. Systematic evaluation of observational methods assessing biomechanical exposures at work. Scand. J. Work. Environ. Health 2010, 36, 3-24. [CrossRef]

11. Lowe, B.D. Accuracy and validity of observational estimates of wrist and forearm posture. Ergonomics 2004, 47, 527-554. [CrossRef]

12. Rhén, I.M.; Forsman, M. Inter- and intra-rater reliability of the OCRA checklist method in video-recorded manual work tasks. Appl. Ergon. 2020, 84, 103025. [CrossRef]

13. Bao, S.; Spielholz, P.; Howard, N.; Silverstein, B. Quantifying repetitive hand activityfor epidemiological research on musculoskeletal disorders-part I: Individual exposure assessment. Ergonomics 2007, 49, 361-380. [CrossRef] [PubMed]

14. Yang, L.; Lu, K.; Diaz-Olivares, J.A.; Seoane, F.; Lindecrantz, K.; Forsman, M.; Abtahi, F.; Eklund, J.A. Towards Smart Work Clothing for Automatic Risk Assessment of Physical Workload. IEEE Access 2018, 6, 40059-40072. [CrossRef]

15. Forsman, M. The search for practical and reliable observational or technical risk assessment methods to be used in prevention of musculoskeletal disorders. Agron. Res. 2017, 15, 680-686. 
16. Hansson, G.Å.; Balogh, I.; Byström, J.U.; Ohlsson, K.; Nordander, C.; Asterland, P.; Sjölander, S.; Rylander, L.; Winkel, J.; Skerfving, S.; et al. Questionnaire versus direct technical measurements in assessing postures and movements of the head, upper back, arms and hands. Scand. J. Work. Environ. Health 2001, 27, 30-40. [CrossRef]

17. Lim, S.; D'Souza, C. A narrative review on contemporary and emerging uses of inertial sensing in occupational ergonomics. Int. J. Ind. Ergon. 2020, 76, 102937. [CrossRef] [PubMed]

18. Robert-Lachaine, X.; Mecheri, H.; Larue, C.; Plamondon, A. Accuracy and repeatability of single-pose calibration of inertial measurement units for whole-body motion analysis. Gait Posture 2017, 54, 80-86. [CrossRef]

19. Robert-Lachaine, X.; Mecheri, H.; Muller, A.; Larue, C.; Plamondon, A. Validation of a low-cost inertial motion capture system for whole-body motion analysis. J. Biomech. 2020, 99, 109520. [CrossRef]

20. Schall, M.C.; Fethke, N.B.; Chen, H.; Gerr, F. A comparison of instrumentation methods to estimate thoracolumbar motion in field-based occupational studies. Appl. Ergon. 2015, 48, 224-231. [CrossRef]

21. Kim, S.; Nussbaum, M.A. Performance evaluation of a wearable inertial motion capture system for capturing physical exposures during manual material handling tasks. Ergonomics 2013, 56, 314-326. [CrossRef]

22. Fan, X.; Lind, C.M.; Rhen, I.M.; Forsman, M. Effects of sensor types and angular velocity computational methods in field measurements of occupational upper arm and trunk postures and movements. Sensors 2021, 21, 5527. [CrossRef]

23. Seidel, D.H.; Heinrich, K.; Hermanns-Truxius, I.; Ellegast, R.P.; Barrero, L.H.; Rieger, M.A.; Steinhilber, B.; Weber, B. Assessment of work-related hand and elbow workloads using measurement-based TLV for HAL. Appl. Ergon. 2021, 92, 103310. [CrossRef] [PubMed]

24. Schoenmarklin, R.W.; Marras, W.S.; Leurgans, S.E. Industrial wrist motions and incidence of hand/wrist cumulative trauma disorders. Ergonomics 1994, 37, 1449-1459. [CrossRef] [PubMed]

25. Balogh, I.; Arvidsson, I.; Björk, J.; Hansson, G.Å.; Ohlsson, K.; Skerfving, S.; Nordander, C. Work-related neck and upper limb disorders-Quantitative exposure-response relationships adjusted for personal characteristics and psychosocial conditions. $B M C$ Musculoskelet. Disord. 2019, 20, 1-19. [CrossRef] [PubMed]

26. Seidel, D.H.; Ditchen, D.M.; Hoehne-Hückstädt, U.M.; Rieger, M.A.; Steinhilber, B. Quantitative measures of physical risk factors associated with work-related musculoskeletal disorders of the elbow: A systematic review. Int. J. Environ. Res. Public Health 2019, 6, 130. [CrossRef]

27. Nordander, C.; Hansson, G.Å.; Ohlsson, K.; Arvidsson, I.; Balogh, I.; Strömberg, U.; Rittner, R.; Skerfving, S. Exposure-response relationships for work-related neck and shoulder musculoskeletal disorders-Analyses of pooled uniform data sets. Appl. Ergon. 2016, 55, 70-84. [CrossRef] [PubMed]

28. Arvidsson, I.; Dahlqvist, C.; Enquist, H.; Nordander, C. mAction Levels for the Prevention of Work-Related Musculoskeletal Disorders in the Neck and Upper Extremities: A Proposal. Ann. Work. Expo. Health 2021, 65, 741-747. [CrossRef]

29. Balogh, I.; Ohlsson, K.; Nordander, C.; Skerfving, S.; Hansson, G.Å. Precision of measurements of physical workload during standardized manual handling part III: Goniometry of the wrists. J. Electromyogr. Kinesiol. 2009, 19, 1005-1012. [CrossRef]

30. Wirth, M.A.; Fischer, G.; Verdú, J.; Reissner, L.; Balocco, S.; Calcagni, M. Comparison of a New Inertial Sensor Based System with an Optoelectronic Motion Capture System for Motion Analysis of Healthy Human Wrist Joints. Sensors 2019, 19, 5297. [CrossRef]

31. Lind, C.M.; Sandsjö, L.; Mahdavian, N.; Högberg, D.; Hanson, L.; Olivares, J.A.D.; Yang, L.; Forsman, M. Prevention of Work: Related Musculoskeletal Disorders Using Smart Workwear-The Smart Workwear Consortium. Adv. Intell. Syst. Comput. 2018, 876, 477-483. [CrossRef]

32. Yang, L.; Grooten, W.J.A.; Forsman, M. An iPhone application for upper arm posture and movement measurements. Appl. Ergon. 2017, 65, 492-500. [CrossRef]

33. Chen, H.; Schall, M.C.; Fethke, N.B. Measuring upper arm elevation using an inertial measurement unit: An exploration of sensor fusion algorithms and gyroscope models. Appl. Ergon. 2020, 89, 103187. [CrossRef] [PubMed]

34. Robert-Lachaine, X.; Mecheri, H.; Larue, C.; Plamondon, A. Effect of local magnetic field disturbances on inertial measurement units accuracy. Appl. Ergon. 2017, 63, 123-132. [CrossRef] [PubMed]

35. Hansson, G.-Å.; Balogh, I.; Ohlsson, K.; Rylander, L.; Skerfving, S. Goniometer measurement and computer analysis of wrist angles and movements applied to occupational repetitive work. J. Electromyogr. Kinesiol. 1996, 6, 23-35. [CrossRef]

36. Li, Z.M.; Kuxhaus, L.; Fisk, J.A.; Christophel, T.H. Coupling between wrist flexion-extension and radial-ulnar deviation. Clin. Biomech. 2005, 20, 177-183. [CrossRef] [PubMed]

37. Hansson, G. $\AA$.; Balogh, I.; Ohlsson, K.; Skerfving, S. Measurements of wrist and forearm positions and movements: Effect of, and compensation for, goniometer crosstalk. J. Electromyogr. Kinesiol. 2004, 14, 355-367. [CrossRef] [PubMed]

38. Jonsson, P.; Johnson, P.W. Comparison of measurement accuracy between two types of wrist goniometer systems. Appl. Ergon. 2001, 32, 599-607. [CrossRef]

39. Buchholz, B.; Wellman, H. Practical operation of a biaxial goniometer at the wrist joint. Hum. Factors 1997, 39, 119-129. [CrossRef]

40. Arvidsson, I.; Åkesson, I.; Hansson, G.Å. Wrist movements among females in a repetitive, non-forceful work. Appl. Ergon. 2003, 34, 309-316. [CrossRef] 